\title{
HPV-Mediated (p16-Positive) Oropharyngeal Cancer TNM Finding v8
}

National Cancer Institute

\section{Source}

National Cancer Institute. HPV-Mediated (p16-Positive) Oropharyngeal Cancer TNM

Finding v8. NCI Thesaurus. Code C132858.

A finding about one or more characteristics of HPV-mediated (p16-positive) oropharyngeal cancer, following the rules of the TNM AJCC v8 classification system. Oropharyngeal cancers which are p16-negative are staged according to the classification for oropharynx (p16-negative) and hypopharynx cancers. (from AJCC 8th Ed.) 